\title{
Conception actuelle des vidanges de fond des barrages d'EDF
}

\author{
J.M. Tinland \\ Electricité de France \\ Service de la Production hydraulique
}

Les considérations suivantes sur les vidanges de fond sont le reflet des enseignements tirés du retour d'expérience des ouvrages en service à EDF.

\section{Rôle des vidanges de fond des barrages}

\section{Rôle fondamental}

"Permettre la vidange totale ou partielle d'une retenue » dans le but de satisfaire à différents besoins comme : assurer la sécurité en cas de problème sur l'ouvrage, rendre possible la visite du parement amont (visites décennales) ou tout simplement maîtriser le niveau du plan d'eau sous le niveau minimal d'exploitation pour réaliser les travaux d'entretien sur des ouvrages comme les prises d'eau.

Nous avons l'habitude d'estimer que la vidange de fond (ou tout organe jouant ce rôle) doit permettre dans l'hypothèse d'apports nuls et sans recours aux autres ouvrages (ouvrage évacuateur de crue-usine) de faire baisser la poussée hydrostatique de moitié en moins de 8 jours, de réaliser la vidange totale de la retenue en moins de 21 jours.

Ces règles interviennent sur le dimensionnement de l'ouvrage et sont appliquées dans le cas général. Des cas particuliers d'ouvrages peuvent nécessiter de déroger à ces règles, par exemple en limitant le débit à la valeur supportable par la vallée à l'aval.

\section{Rôle auxiliaire}

Une des phases essentielles de la vie d'un barrage est la période de première mise en eau qui exige de la part du maître d'ouvrage une totale maîtrise du niveau du plan d'eau pour assurer la sécurité.

La vidange de fond, si elle est suffisamment dimensionnée, peut servir d'organe de sécurité permettant de contrôler la première mise en charge du barrage et d'abaisser rapidement le niveau de la retenue si des anomalies apparaissent dans le comportement de l'ouvrage. Ce rôle, pour qu'il soit efficace, peut requérir des débits de vidange assez élevés dont il convient de fixer les valeurs en tenant compte des possibilités d'écoulement à l'aval.

\section{Rôle exceptionnel}

Les vidanges de fond peuvent, dans certains cas, être incluses dans le dispositif d'évacuation des crues. Dans ce cas, elles doivent être conçues et équipées telles que des évacuateurs de crues et la fonction de vidange ne devient plus alors qu'un corollaire mineur et occasionnel de la vocation principale. Il ne faut pas perdre de vue les différences de fonctionnement qui se répercuteront directement sur la conception :

- la fonction vidange induit un fonctionnement exceptionnel de durée limitée, à charge rapidement décroissante, - la fonction évacuateur de crues implique des fonctionnements répétitifs, éventuellement de longue durée, en exploitation normale, à pleine charge et à débit souvent variable.

On demandera donc à ces matériels, en plus des conditions de fonctionnement particuliers, plus de robustesse, plus de résistance aux vibrations, etc...

Cette disposition constitue un moyen de lutte contre l'envasement par l'évacuation des courants de densité.

\section{Remarque}

Il importe de se convaincre que les vidanges de fond n'ont pas pour but d'assurer des restitutions de débits réservés qui ne correspondent pas à la plage de débit d'une vidange de fond. Cette disposition nécessiterait des types de vannes spéciales (pointeau - jet creux) beaucoup trop sujettes au colmatage.

\section{Problèmes d'exploitation des vidanges de fond}

\subsection{L'alluvionnement - le charriage}

Il s'agit de loin du problème le plus important rencontré par les exploitants. Il se présente sous les aspects suivants :

- remblaiement complet de la prise d'eau, quelquefois sous plusieurs dizaines de mètres; 
- vannes rendues complètement indisponibles par colmatage (efforts de manœuvre);

- vannes non adaptées au charriage d'éléments naturels (limons, arbres, souches) ou artificiels (pneus, plastiques, bidons, etc...) par leur conception (vannes, pointeau, vannes à jet creux).

Si l'on veut que les vidanges de fond soient des organes de sécurité, il faut résoudre ces problèmes et donner confiance à l'exploitant dans la manœuvre de ces vannes.

\subsection{Les vibrations}

Les vidanges de fond qui doivent fonctionner sous la plus forte charge sont le siège d'écoulements à grande vitesse $(15-25 \mathrm{~m} / \mathrm{s})$ qui sont de nature à provoquer des vibrations et des phénomènes de résonances.

Deux dispositifs participent au moins à l'amélioration de ces phénomènes :

- un surdimensionnement des matériels d'autant plus important quand les vidanges sont utilisées en évacuateurs de crues ou pour faire transiter les courants de densité ; - la conception de conduits lisses favorisant l'écoulement de l'eau sans obstacle même minime.

\subsection{L'érosion du lit aval}

L'eau évacuée par une vidange de fond sort à grande vitesse, donc avec une grande énergie. Le lit de la rivière devra être susceptible de supporter l'énergie du jet sans érosion ou destruction. Il est souvent nécessaire de réaliser un modèle réduit hydraulique pour mieux étudier tous ces problèmes.

\section{Dispositions générales constructives}

\subsection{Implantation}

Concernant la cote d'implantation des vidanges de fond, la notion de culot ou tranche morte est admise, car l'expérience a montré que dans les zones à fort alluvionnement, il était impossible ou invraisemblable de vouloir maintenir cette partie de la retenue dégagée. L'action des vidanges s'exerce immédiatement à l'amont du barrage dans un cône réduit où les vitesses sont suffisantes pour remettre en suspension les matériaux et l'on constate que l'érosion devient active dès le passage en régime torrentiel de l'eau en fin de vidange.

Dans ces conditions, au concept du niveau le plus bas pour une vidange, il faut adjoindre le niveau le plus haut pour lequel l'engravement de la retenue aurait des répercussions sur le bon fonctionnement des autres ouvrages comme les prises d'eau et les évacuateurs de crues.

Les notions de seconde vidange (en sécurité de la principale) sous la forme d'un conduit à une altitude supérieure ou de l'aménagement de dérivation provisoire avec des fonds explosibles sont considérées comme secondaires et marginales. Elles peuvent être utiles pour des travaux de gros entretien mais en aucun cas elles ne doivent être comptabilisées dans le système officiel.

Dès lors, il est recommandé de concevoir les vidanges de fond pour être assuré de leur bon fonctionnement dans toutes les conditions de service.

\subsection{Conception générale}

Les vidanges de fond comprennent en général 4 éléments constitutifs :

- une grille de protection à l'amont,

- une vanne de garde,

- un conduit d'évacuation,

- une vanne aval de réglage du débit accompagnée d'une éventuelle cuiller de dispersion du jet.

La distance entre les deux vannes est supérieure ou égale à 6 mètres pour éviter qu'un corps transporté (arbre ou autre objet) puisse condamner le fonctionnement des deux vannes simultanément. Le diamètre du conduit doit être supérieur ou égal à 2 mètres.

Les critères mentionnés représentent des limites générales à respecter.

\subsection{Caractéristiques particulières des vidanges de fond}

\subsubsection{La grille de protection}

On entend par grille de protection, l'ensemble de l'ouvrage dont la mission est de protéger le conduit de la vidange de fond contre l'obstruction par les produits de charriage et garantir son alimentation.

Dimensionnement de la grille: Les orifices de passage seront tels que tout corps qui les traverse doit transiter sans problème dans le conduit de vidange. De plus, pour limiter leur disposition au colmatage, ils devront être les plus grands acceptables.

En définitive, la section de l'orifice de passage peut être choisie entre $60 \%$ et $90 \%$ de la section du conduit de vidange, aucune dimension de l'orifice ne devrait être plus grande que le diamètre de la vidange.

Malgré le dimensionnement large des orifices de passage de la grille, il existe toujours une possibilité de colmatage par des transports solides de grand volume comme les arbres, souches... Il reste utile de concevoir la tour de prise d'eau avec une très grande surface totale d'entrée d'eau.

La structure est calculée à la rupture pour une hypothèse d'obstruction totale.

\subsubsection{Le conduit de vidange}

Malgré l'existence de l'ouvrage de protection amont, il faut permettre le passage accidentel de corps étrangers (troncs d'arbres, pneus, souches, ...) de grande dimension et le passage permanent des matériaux de charriage habituels.

A cet effet, il convient d'adopter, dans tous les cas, des conduits de vidange dont le diamètre ne descende pas en 
dessous de 2 mètres. En outre, leur tracé sera le plus court et aussi rectiligne que possible. La convergence pour la vanne aval sera réduite au minimum.

D'une façon générale, en exploitation, le conduit est en charge, vanne aval fermée, vanne amont ouverte et en conséquence le conduit de vidange est blindé métallique.

\subsubsection{La vanne de garde amont}

La vanne amont sera conçue pour suppléer la vanne aval dans le cas de mauvais fonctionnement de celle-ci en fermeture et ainsi être capable de couper le débit maximal sous la plus forte charge. En principe, cette vanne à effacement total fonctionne par tout ou rien.

Les vannes de vidange de fond sont des matériels soumis à des efforts violents. Il est donc nécessaire d'adopter des matériels robustes, rustiques et exigeant l'entretien minimal.

Il est impératif, lorsque l'on conçoit une vidange, de respecter le principe de la vanne aval fermée assurant l'étanchéité et le réglage du débit, et de la vanne de garde amont rustique, ouverte en exploitation normale.

\subsubsection{La vanne de réglage aval}

Située à une distance au moins égale à 6 mètres de la vanne amont, la vanne aval est une vanne de réglage du débit, en conséquence, elle est à effacement total du type segment. Pour les vidanges de faibles caractéristiques, elle pourra être à la rigueur du type vanne plate à glissières.

La définition de ces ouvrages aval (cuiller, etc...) rend nécessaire leur étude sur modèle réduit hydraulique. Il faut se rappeler que les phénomènes hydrauliques à grande vitesse ne s'appréhendent pas facilement par le calcul et que ces essais représentent encore le meilleur moyen d'effectuer une approche de la réalité.

\section{Exploitation des ouvrages de vidange}

L'objectif des considérations exposées aux chapitres précédents est essentiellement de mettre à la disposition de l'exploitant des organes de vidange qui lui inspirent confiance, à la fois par leur conception rationnelle et la qualité du matériel.

Ainsi, le personnel responsable sur place, à peu près affranchi du risque qu'il redoute le plus (ne plus pouvoir refermer une vanne de vidange obstruée au cours d'un essai de fonctionnement), sera-t-il enclin à pratiquer plus fréquemment des manœuvres périodiques, ce qui constitue en fait le meilleur moyen d'éviter l'obstruction de l'ouvrage de vidange.

Ces chasses doivent être exécutées régulièrement (une ou deux fois par an) dans le cas de retenues aux eaux fortement chargées, afin d'assurer au mieux l'évacuation des matériaux sans laisser aux dépôts le temps de s'accumuler et de se consolider au voisinage des orifices de vidange.

L'ouverture des vannes sera maximale dans la limite des débits admissibles à l'aval (il faut en effet tenir compte du débit que peut supporter sans dommage le lit aval, etc...).

En particulier, il est conseillé de multiplier les chasses au cours des premières années d'exploitation des retenues et de les pratiquer, si possible, au moment des crues où les charriages sont les plus importants.

En aucun cas des notions économiques (pertes d'énergie) ne peuvent faire déroger à l'application des consignes d'exploitation d'essai des vidanges.

\section{Conclusion}

Le retour d'expérience pratiqué sur les ouvrages EDF nous montre que l'application des dispositions ci-dessus explicitées permet une exploitation correcte des vidanges de fond dans de très bonnes conditions de sécurité pour l'ouvrage.

Il reste entendu que les choix à effectuer dans la conception et l'exploitation des vidanges de fond doivent être conduits dans l'esprit de ces dispositions en tenant compte des spécificités propres à chaque barrage. 


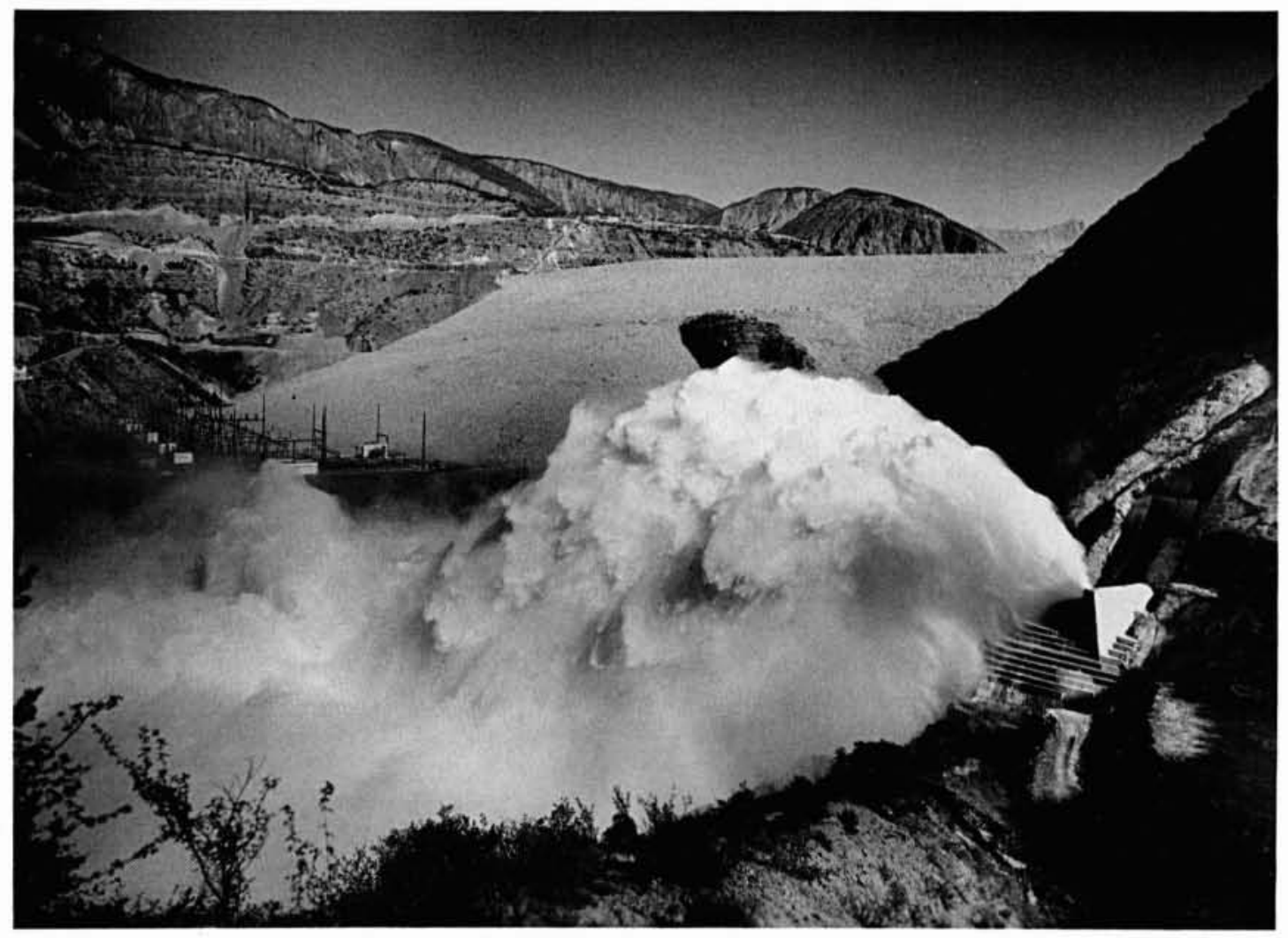

Serre-Ponçon : évacuateur de crues en fonctionnement

Photo H. Baranger 